\title{
Evolution of magnetic fields in the IGM: kinetic MHD turbulence
}

\author{
Reinaldo S. de Lima ${ }^{1}$, E. M. de Gouveia Dal Pino ${ }^{1}$, A. Lazarian ${ }^{2}$ \\ and D. Falceta-Gonçalves ${ }^{3}$ \\ ${ }^{1}$ Instituto de Astronomia, Geofísica e C. Atmosféricas, Universidade de São Paulo, Brazil \\ email: rlima@astro.iag.usp.br, dalpino@astro.iag.usp.br \\ ${ }^{2}$ Núcleo de Astrofísica Teórica, Universidade Cruzeiro do Sul, Brazil \\ ${ }^{3}$ Astronomy Department, University of Wisconsin, USA
}

\begin{abstract}
In this work, we present 3D MHD simulations of non-helical, forced turbulence, with an anisotropic thermal pressure with respect to the orientation of the local magnetic field. Such anisotropy arises when the plasma is weakly collisional, i.e., when the Larmor frequency is much greater than the ion-ion collision frequency. In this Kinetic MHD regime (KMHD), there are instabilities that give rise to fast growing magnetic fluctuations in the smallest scales. The plasma that fills the intergalactic and intracluster media has small density $\left(n \sim 10^{-3} \mathrm{~cm}^{-3}\right)$, hence the effects of these instabilities could be important in the turbulent amplification of the magnetic fields there. In order to study the KMHD turbulence, we have performed 3D numerical simulations employing a godunov-MHD code (e.g., Kowal, Lazarian \& Beresnyak 2007; FalcetaGonçalves, Lazarian \& Kowal 2008). The power spectrum of the velocity and magnetic fields were calculated for two cases: when there is a pre-existing mean magnetic field, and when there is only an initial weak magnetic field.
\end{abstract}

Keywords. Magnetic fields - plasmas - intergalactic medium

\section{Kinetic MHD description}

Due the low density, an MHD description of the IGM and ICM is not adequate: the ions Larmor radius $r_{L}$ is much smaller than its mean free path $\lambda$. For instance, in the Hydra A cluster, $r_{L} \sim 10^{5} \mathrm{~km}$ and $\lambda \sim 10^{15} \mathrm{~km}$ (Ensslin \& Vogt 2006). A collisionless plasma can be described by an MHD set of equations under the kinematic approximation (the KMHD equations), where the scalar pressure in the ideal MHD equations is replaced by the following tensor:

$$
P_{i j}=p_{\perp} \delta_{i j}+\left(p_{\|}-p_{\perp}\right) B_{i} B_{j} / \mathbf{B}^{2}
$$

where $p_{\|}$and $p_{\perp}$ are the components of the pressure in the directions parallel and perpendicular to $\mathbf{B}$. The standard approach for each pressure component is the double-adiabatic law:

$$
\frac{d}{d t}\left(\frac{p_{\perp}}{\rho B}\right)=0 \quad \frac{d}{d t}\left(\frac{p_{\|} B^{2}}{\rho^{3}}\right)=0
$$

However, in the preliminary numerical simulations shown below, we have adopted an isothermal description for the pressure components in each direction, with sound velocities $c_{\text {perp }}$ and $c_{\text {par }}$.

In all simulations, the turbulence is forced in a $3 \mathrm{D}$ periodic box (with $128^{3}$ resolution) by a random, non-helical, solenoidal force acting around the forcing wave number $k_{f}$. The velocity of the forcing scale is $u_{f} \sim 1$. 
Turbulence in the presence of an external magnetic field: In these simulations, the external magnetic field $\mathbf{B}_{\text {ext }}$ is uniform with Alfénic Mach number $M_{A}=1$ (see Fig. 1).
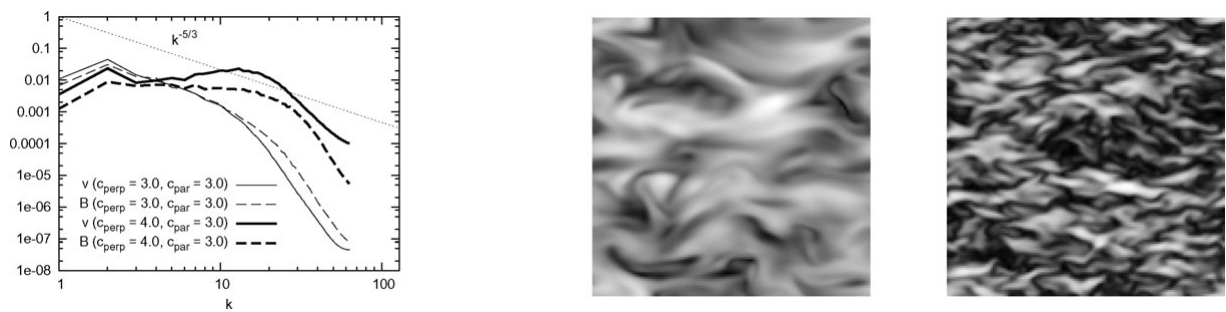

Figure 1. Left: power spectrum of the velocity and magnetic fields for a collisional MHD simulation with $c_{\text {perp }}=c_{\text {par }}=3.0(\mathrm{MHD})$ and for a collisionless kinetic MHD simulation $c_{\text {perp }}=4.0, c_{\text {par }}=3.0(\mathrm{KMHD})$. The images in the right show $|\mathbf{B}|$ in the central slices of the cubes, with horizontal $\mathbf{B}_{e x t}$ (middle: MHD, right: KMHD). In these simulations $k_{f}=2.5$.

Growth of a weak initial magnetic field: For different degrees of pressure anisotropy and the same initial weak magnetic field (see Fig. 2).
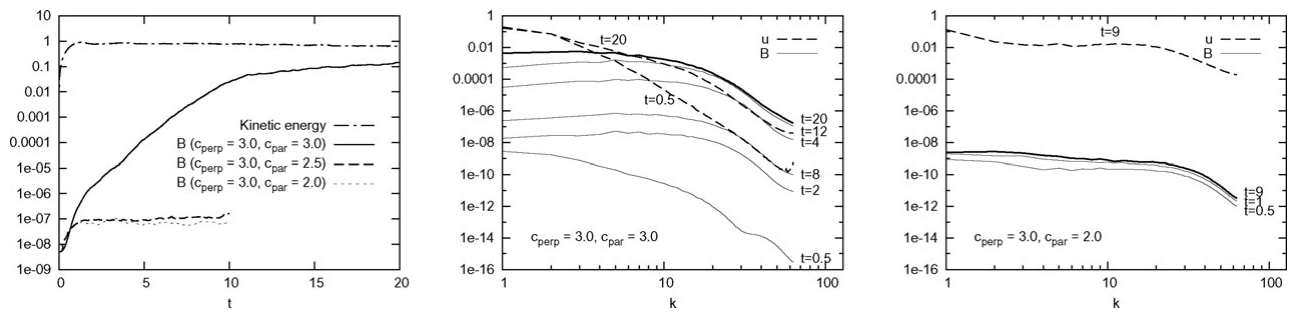

Figure 2. Left: Magnetic energy evolution. Middle and right: magnetic power spectrum evolution for the MHD case and KMHD case, respectively. In these simulations $k_{f}=1.5$.

The instabilities that develop due to the anisotropic pressure accumulate energy at the smallest scales (limited by the numerical dissipation), changing the inclination of the spectrum in the inertial range. The small-scale fluctuations of the fields due to these instabilities give rise to a more "wrinkled" field distribution in the KMHD case.

In the simulations with anisotropic pressure, the dynamo action seems to be inhibited. This issue however, still requires deeper investigation with simulations spanning a larger parameter space.

Further simulations including a double-adiabatic law must be performed in order to study the turbulence development with spontaneously raised anisotropic pressures.

\section{Acknowledgements}

This work is partially suported by the Brazilian agency Fundação de Amparo à Pesquisa do Estado de São Paulo (FAPESP).

\section{References}

Ensslin, T. A. \& C. Vogt, C. 2006, A\&A 453, 447

Falceta-Gonçalves, D., Lazarian A., \& Kowal, G. 2008, ApJ 679, 537

Kowal, G., Lazarian, A., \& Beresnyak, A. 2007, ApJ 658, 423 